\title{
ON A UNIQUENESS THEOREM OF H. UEDA
}

\author{
IndRajit LahiRi AND Pulak SAHoO
}

AbStRact. We prove a uniqueness theorem for meromorphic functions sharing three weighted values which improves some existing results.

\section{Introduction, definitions and results}

Let $f$ and $g$ be two non-constant meromorphic functions defined in the open complex plane $\mathbb{C}$. For $a \in \mathbb{C} \cup\{\infty\}$ we say that $f, g$ share the value a CM (counting multiplicities) if $f, g$ have the same $a$-points with the same multiplicity and we say that $f, g$ share the value $a$ IM (ignoring multiplicities) if we do not consider the multiplicities. For standard definitions and notations of the value distribution theory we refer the reader to [5].

In 1980, H. Ueda [18] proved the following result.

Theorem A ([18]). Let $f$ and $g$ be two distinct non-constant entire functions sharing $0,1 C M$ and let $a(\neq 0,1)$ be a finite complex number. If a is lacunary for $f$, then $1-a$ is lacunary for $g$ and $(f-a)(g+a-1) \equiv a(1-a)$.

After the publication of [18] a great deal of work has been done on Theorem A (cf. $[1,2,3,6,10,12,20,21])$. In 1995 H. X. Yi [21] extended Theorem A to meromorphic functions and proved the following result.

Theorem B ([21]). Let $f$ and $g$ be two distinct non-constant meromorphic functions sharing $0,1, \infty C M$ and $a(\neq 0,1)$ be a finite complex number. If $N(r, a ; f) \neq T(r, f)+S(r, f)$, then one of the following holds:

(i) $(f-a)(g+a-1) \equiv a(1-a)$,

(ii) $f+(a-1) g \equiv a$,

(iii) $f \equiv a g$.

We now require the following definition.

Definition 1.1. Let $p$ be a positive integer and $b \in \mathbb{C} \cup\{\infty\}$. Then by $N(r, b ; f \mid \leq p)$ we denote the counting function of those $b$-points of $f$ (counted

Received June 21, 2008

2000 Mathematics Subject Classification. 30D35.

Key words and phrases. meromorphic functions, weighted sharing, uniqueness. 
with multiplicities) whose multiplicities are not greater than $p$. By $\bar{N}(r, b ; f \mid \leq$ $p$ ) we denote the corresponding reduced counting function.

In an analogous manner we define $N(r, b ; f \mid \geq p)$ and $\bar{N}(r, b ; f \mid \geq p)$.

In 2004, X. M. Li and H. X. Yi [16] further worked on Theorem B and proved the following result.

Theorem C ([16]). Let $f$ and $g$ be two nonconstant meromorphic functions sharing $0,1, \infty C M$. If there exists a number $a(\neq 0,1, \infty)$ such that a is not a Picard exceptional value of $f$ and

$$
N(r, a ; f \mid \leq 1) \neq T(r, f)+S(r, f),
$$

then

$$
N(r, a ; f \mid \leq 1)=\frac{p-2}{p} T(r, f)+S(r, f)
$$

and one of the following cases holds:

(i) $f=\frac{e^{(p+1) \gamma}-1}{e^{s \gamma}-1}, g=\frac{e^{-(p+1) \gamma}-1}{e^{-s \gamma}-1}$ with $\frac{(a-1)^{p+1-s}}{a^{p+1}}=\frac{s^{s}(p+1-s)^{p+1-s}}{(p+1)^{p+1}}$ and $a \neq$ $\frac{p+1}{s}$

(ii) $f=\frac{e^{s \gamma}-1}{e^{(p+1) \gamma}-1}, g=\frac{e^{-s \gamma}-1}{e^{-(p+1) \gamma}-1}$ with $a^{s}(1-a)^{p+1-s}=\frac{s^{s}(p+1-s)^{p+1-s}}{(p+1)^{p+1}}$ and $a \neq \frac{s}{p+1}$

(iii) $f=\frac{e^{s \gamma}-1}{e^{-(p+1-s) \gamma}-1}, g=\frac{e^{-s \gamma}-1}{e^{(p+1-s) \gamma}-1}$ with $\frac{(-a)^{s}}{(1-a)^{p+1}}=\frac{s^{s}(p+1-s)^{p+1-s}}{(p+1)^{p+1}}$ and $a \neq-\frac{s}{p+1-s}$

(iv) $f=\frac{e^{p \gamma}-1}{\lambda e^{s \gamma}-1}, g=\frac{e^{-p \gamma}-1}{\frac{1}{\lambda} e^{-s \gamma}-1}$ with $\lambda^{p} \neq 0,1$ and $\frac{(a-1)^{p-s}}{\lambda^{p} a^{p}}=\frac{s^{s}(p-s)^{p-s}}{p^{p}}$;

(v) $f=\frac{e^{s \gamma}-1}{\lambda e^{p \gamma}-1}, g=\frac{e^{-s \gamma}-1}{\frac{1}{\lambda} e^{-p \gamma}-1}$ with $\lambda^{s} \neq 0,1$ and $\lambda^{s} a^{s}(1-a)^{p-s}=\frac{s^{s}(p-s)^{p-s}}{p^{p}}$;

(vi) $f=\frac{e^{s \gamma}-1}{\lambda e^{-(p-s) \gamma}-1}, g=\frac{e^{-s \gamma}-1}{\frac{1}{\lambda} e^{(p-s) \gamma}-1}$ with $\lambda^{s} \neq 0,1$ and $\frac{(-\lambda a)^{s}}{(1-a)^{p}}=\frac{s^{s}(p-s)^{p-s}}{p^{p}}$, where $\gamma$ is a nonconstant entire function, $s$ and $p(\geq 2)$ are positive integers such that $s$ and $p+1$ are relatively prime and $1 \leq s \leq p$ in (i), (ii) and (iii), $s$ and $p$ are relatively prime and $1 \leq s \leq p-1$ in (iv), (v) and (vi).

Following two examples show that in Theorem B the sharing of 0 and 1 can not be relaxed from CM to IM.

Example 1.1 ([10]). Let $f=e^{z}-1$ and $g=\left(e^{z}-1\right)^{2}$ and $a=-1$. Then $f$, $g$ share 0 IM and $1, \infty$ CM. Also $N(r, a ; f) \equiv 0$ but Theorem B does not hold.

Example $1.2([10])$. Let $f=e^{z}-2, g=e^{z}\left(2-e^{z}\right)$ and $a=2$. Then $f, g$ share $1 \mathrm{IM}$ and $0, \infty \mathrm{CM}$. Also $N(r, a ; f) \equiv 0$ but Theorem B does not hold.

In view of above examples attempts have been made in [3] and [10] to improve Theorem B by relaxing the nature of value sharing. This was done with the aid of a notion of weighted value sharing, which measures how close a shared value is to be shared CM or to be shared IM. 
Definition $1.2([7,8])$. Let $k$ be a nonnegative integer or infinity. For $a \in$ $\mathbb{C} \cup\{\infty\}$ we denote by $E_{k}(a ; f)$ the set of all $a$-points of $f$, where an $a$-point of multiplicity $m$ is counted $m$ times if $m \leq k$ and $k+1$ times if $m>k$. If $E_{k}(a ; f)=E_{k}(a ; g)$, we say that $f, g$ share the value $a$ with weight $k$.

The definition implies that if $f, g$ share a value $a$ with weight $k$, then $z_{o}$ is a zero of $f-a$ with multiplicity $m(\leq k)$ if and only if it is a zero of $g-a$ with multiplicity $m(\leq k)$ and $z_{o}$ is a zero of $f-a$ with multiplicity $m(>k)$ if and only if it is a zero of $g-a$ with multiplicity $n(>k)$, where $m$ is not necessarily equal to $n$.

We write $f, g$ share $(a, k)$ to mean that $f, g$ share the value $a$ with weight $k$. Clearly if $f, g$ share $(a, k)$, then $f, g$ share $(a, p)$ for all integers $p, 0 \leq p<k$. Also we note that $f, g$ share a value $a$ IM or CM if and only if $f, g$ share $(a, 0)$ or $(a, \infty)$ respectively.

In order to state the next results we require the following definitions.

Definition 1.3. A meromorphic function $a$ is called small with respect to $f$ if $T(r, a)=S(r, f)$.

Definition 1.4. We denote by $\mathfrak{M}_{f, g}$ the collection of all meromorphic functions which are small with respect to $f$ and $g$.

Definition 1.5. For a small function $a$, we define by $N(r, a ; f), \bar{N}(r, a ; f)$, $N(r, a ; f \mid \leq p), \bar{N}(r, a ; f \mid \leq p), N(r, a ; f \mid \geq p), \bar{N}(r, a ; f \mid \geq p)$ etc. the corresponding counting functions of the zeros of $f-a$.

Definition 1.6. An entire function $f$ is said to be an exponential function if $f=\exp (\beta)$ for some entire function $\beta$.

We now state two results which improve Theorem B.

Theorem D ([3]). Let $f$ and $g$ be two distinct non-constant meromorphic functions sharing $\left(a_{1}, 1\right),\left(a_{2}, \infty\right)$ and $\left(a_{3}, \infty\right)$, where $\left\{a_{1}, a_{2}, a_{3}\right\}=\{0,1, \infty\}$. If for a complex number $a(\neq 0,1, \infty), N(r, a ; f \mid \leq 2) \neq T(r, f)+S(r, f)$, then the conclusion of Theorem $B$ holds.

Theorem E (cf. Remark 1 [10]). Let $f$ and $g$ be two distinct non-constant meromorphic functions sharing $(0,1),(1, m)$ and $(\infty, k)$, where $(m-1)(k-$ 1) $>4$. If $a(\neq 0,1)$ is a finite complex number such that $N(r, a ; f \mid \leq 2) \neq$ $T(r, f)+S(r, f)$, then the conclusion of Theorem B holds.

In 2005 T. C. Alzahary considered the problem of replacing the constant $a(\neq 0,1, \infty)$ in Theorem B by a small function $a=a(z)(\not \equiv 0,1, \infty)$. He proved the following result.

Theorem $\mathbf{F}([1])$. Let $f$ and $g$ be two distinct non-constant meromorphic functions sharing $(0,1),(1, \infty),(\infty, \infty)$. If $N(r, a ; f) \neq T(r, f)+S(r, f)$ for some $a=a(z)(\not \equiv 0,1, \infty) \in \mathfrak{M}_{f, g}$, then there exists a non-constant meromorphic function $\gamma$, such that $f$ and $g$ satisfy one of the following three possibilities: 
(i) $f=a\left(1-e^{-\gamma}\right)$ and $g=(a-1)\left(e^{\gamma}-1\right)$, where $\gamma$ is an entire function and $1-\frac{1}{a}$ is an exponential function if and only if $f, g$ share $(0, \infty)(1, \infty)$ and $(\infty, \infty)$;

(ii) $f=\frac{a}{1-\gamma(1-a)}$ and $g=\frac{a}{\gamma^{-1}-(1-a)}$, where $1-a$ is an exponential function and $\bar{N}(r, \infty ; \gamma)+\bar{N}(r, 0 ; \gamma)=S(r, g)$ and $\gamma$ is an exponential function if and only if $f, g$ share $(0, \infty)(1, \infty)$ and $(\infty, \infty)$;

(iii) $f=\frac{a\left(1-e^{\gamma}\right)}{1-a e^{\gamma}}$ and $g=\frac{e^{\gamma}-1}{a e^{\gamma}-1}$, where $\gamma$ is an entire function. Also $a$ is an exponential function if and only if $f, g$ share $(0, \infty)(1, \infty)$ and $(\infty, \infty)$.

Following result is an improvement of Theorem F.

Theorem G ([12]). Let $f, g$ be two distinct meromorphic functions sharing $(0,1),(1,2),(\infty, \infty)$. If $N(r, a ; f \mid \leq 2) \neq T(r, f)+S(r, f)$ for some $a(\not \equiv$ $0,1, \infty) \in \mathfrak{M}_{f, g}$, then one of the following holds:

(i) $f=a\left(1-\gamma^{-1}\right)$ and $g=(a-1)(\gamma-1)$, where $\gamma$ is a meromorphic function with $\bar{N}(r, 0 ; \gamma)+\bar{N}(r, \infty ; \gamma)=S(r, f)$. If $f, g$ share $(1, \infty)$ and $(\infty, \infty)$, then $\gamma$ is an exponential function. Further $f, g$ share $(0, \infty),(1, \infty)$, $(\infty, \infty)$ if and only if $1-\frac{1}{a}$ is an exponential function.

(ii) $f=\frac{a}{1-\gamma(1-a)}$ and $g=\frac{a}{\gamma^{-1}-(1-a)}$, where $\gamma$ is a meromorphic function with $\bar{N}(r, 0 ; \gamma)+\bar{N}(r, \infty ; \gamma)=S(r, f)$. If $f, g$ share $(0, \infty)$ and $(\infty, \infty)$, then $\gamma$ is an exponential function. Further $f, g$ share $(1, \infty),(\infty, \infty)$ if and only if $1-a$ is an exponential function.

(iii) $f=\frac{a(\gamma-1)}{a \gamma-1}$ and $g=\frac{\gamma-1}{a \gamma-1}$, where $\gamma$ is a meromorphic function with $\bar{N}(r, 0 ; \gamma)+\bar{N}(r, \infty ; \gamma)=S(r, f)$. If $f, g$ share $(1, \infty)$ and $(\infty, \infty)$, then $\gamma$ is an exponential function. Further $f, g$ share $(0, \infty),(\infty, \infty)$ if and only if $a$ is an exponential function.

Recently T. C. Alzahary [2] proved the following improved version of Theorem B.

Theorem $\mathbf{H}([2])$. Let $f$ and $g$ be two distinct non-constant meromorphic functions sharing $\left(0, k_{1}\right),\left(1, k_{2}\right),\left(\infty, k_{3}\right)$, where $k_{1} k_{2} k_{3}>k_{1}+k_{2}+k_{3}+2$. If $N(r, a ; f \mid \leq 2) \neq T(r, f)+S(r, f)$ for some $a(\not \equiv 0,1, \infty) \in \mathfrak{M}_{f, g}$, then one of the following holds:

(i) $(f-a)(g+a-1) \equiv a(1-a)$,

(ii) $f+(a-1) g \equiv a$,

(iii) $f \equiv a g$.

Considering $f=e^{z}\left(1-e^{z}\right), g=e^{-z}\left(1-e^{-z}\right)$ and $a=\frac{1}{4}$ we see that in the above results the hypothesis $N(r, a ; f \mid \leq 2) \neq T(r, f)+S(r, f)$ can not be relaxed to $N(r, a ; f \mid \leq 1) \neq T(r, f)+S(r, f)$. So it is an interesting problem to investigate the situation when $N(r, a ; f \mid \leq 1) \neq T(r, f)+S(r, f)$. In the paper we deal with this problem and prove the following theorem.

Theorem 1.1. Let $f$ and $g$ be two distinct non-constant meromorphic functions sharing $\left(0, k_{1}\right),\left(1, k_{2}\right),\left(\infty, k_{3}\right)$, where $k_{1} k_{2} k_{3}>k_{1}+k_{2}+k_{3}+2$. If 
$N(r, a ; f \mid \leq 1) \neq T(r, f)+S(r, f)$ for some $a(\not \equiv 0,1, \infty) \in \mathfrak{M}_{f, g}$, then one of the following holds:

(I) $f=a\left(1-\gamma^{-1}\right)$ and $g=(a-1)(\gamma-1)$, where $\gamma$ is a meromorphic function with $\bar{N}(r, 0 ; \gamma)+\bar{N}(r, \infty ; \gamma)=S(r, f)$. If $f, g$ share $(1, \infty)$ and $(\infty, \infty)$, then $\gamma$ is an exponential function. Further $f, g$ share $(0, \infty)$, $(1, \infty),(\infty, \infty)$ if and only if $1-\frac{1}{a}$ is an exponential function. Also $\bar{N}(r, a ; f)=S(r, f)$ and $N(r, a ; f)=N(r, \infty ; \gamma)+S(r, f)$.

(II) $f=\frac{a}{1-\gamma(1-a)}$ and $g=\frac{a}{\gamma^{-1}-(1-a)}$, where $\gamma$ is a meromorphic function with $\bar{N}(r, 0 ; \gamma)+\bar{N}(r, \infty ; \gamma)=S(r, f)$. If $f$, g share $(0, \infty)$ and $(\infty, \infty)$, then $\gamma$ is an exponential function. Further $f, g$ share $(1, \infty),(\infty, \infty)$ if and only if $1-a$ is an exponential function. Also $\bar{N}(r, a ; f)=S(r, f)$ and $N(r, a ; f)=N(r, 0 ; \gamma)+S(r, f)$.

(III) $f=\frac{a(\gamma-1)}{a \gamma-1}$ and $g=\frac{\gamma-1}{a \gamma-1}$, where $\gamma$ is a meromorphic function with $\bar{N}(r, 0 ; \gamma)+\bar{N}(r, \infty ; \gamma)=S(r, f)$. If $f, g$ share $(1, \infty)$ and $(\infty, \infty)$, then $\gamma$ is an exponential function. Further $f, g$ share $(0, \infty),(\infty, \infty)$ if and only if $a$ is an exponential function. Also $\bar{N}(r, a ; f)=S(r, f)$ and $N(r, a ; f)=N(r, 0 ; \gamma)+S(r, f)$.

(IV) $a$ is a constant with $N(r, a ; f \mid \leq 1)=\frac{p-2}{p} T(r, f)+S(r, f)$ and there exists a non-constant exponential function $\gamma$ such that

(i) $f=\frac{\gamma^{(p+1)}-1}{\gamma^{s}-1}, g=\frac{\gamma^{-(p+1)}-1}{\gamma^{-s}-1}$ with $\frac{(a-1)^{p+1-s}}{a^{p+1}}=\frac{s^{s}(p+1-s)^{p+1-s}}{(p+1)^{p+1}}$ and $a \neq \frac{p+1}{s}$

(ii) $f=\frac{\gamma^{s}-1}{\gamma^{(p+1)}-1}, g=\frac{\gamma^{-s}-1}{\gamma^{-(p+1)}-1}$ with $a^{s}(1-a)^{p+1-s}=\frac{s^{s}(p+1-s)^{p+1-s}}{(p+1)^{p+1}}$ and $a \neq \frac{s}{p+1}$

(iii) $f=\frac{\gamma^{s}-1}{\gamma^{-(p+1-s)}-1}, g=\frac{\gamma^{-s}-1}{\gamma^{(p+1-s)}-1}$ with $\frac{(-a)^{s}}{(1-a)^{p+1}}=\frac{s^{s}(p+1-s)^{p+1-s}}{(p+1)^{p+1}}$ and $a \neq-\frac{s}{p+1-s}$

(iv) $f=\frac{\gamma^{p}-1}{\lambda \gamma^{s}-1}, g=\frac{\gamma^{-p}-1}{\frac{1}{\lambda} \gamma^{-s}-1}$ with $\lambda^{p} \neq 0,1$ and $\frac{(a-1)^{p-s}}{\lambda^{p} a^{p}}=\frac{s^{s}(p-s)^{p-s}}{p^{p}}$;

(v) $f=\frac{\gamma^{s}-1}{\lambda \gamma^{p}-1}, g=\frac{\gamma^{-s}-1}{\frac{1}{\lambda} \gamma^{-p}-1}$ with $\lambda^{s} \neq 0,1$ and $\lambda^{s} a^{s}(1-a)^{p-s}=$ $\frac{s^{s}(p-s)^{p-s}}{p^{p}}$;

(vi) $f=\frac{\gamma^{s}-1}{\lambda \gamma^{-(p-s)}-1}, g=\frac{\gamma^{-s}-1}{\frac{1}{\lambda} \gamma^{(p-s)}-1}$ with $\lambda^{s} \neq 0,1$ and $\frac{(-\lambda a)^{s}}{(1-a)^{p}}=$ $\frac{s^{s}(p-s)^{p-s}}{p^{p}}$

where $s$ and $p(\geq 2)$ are positive integers such that $s$ and $p+1$ are relatively prime and $1 \leq s \leq p$ in (i), (ii) and (iii), $s$ and $p$ are relatively prime and $1 \leq s \leq p-1$ in (iv), (v) and (vi).

(V) $a$ is non-constant such that

(i) $f=\frac{(1-a) \gamma^{p}-1}{\frac{1-a}{a} \lambda \gamma^{q}-1}$ and $g=\frac{\frac{1}{1-a} \gamma^{-p}-1}{\frac{a}{(1-a) \lambda} \gamma^{-q}-1}$, where $\gamma$ is a non-constant meromorphic function with $\bar{N}(r, 0 ; \gamma)+\bar{N}(r, \infty ; \gamma)=S(r, f), p$, $q$ are mutually prime distinct positive integers and $\lambda(\neq 0)$ is a 
constant satisfying

$$
\left(\frac{1}{q-p}\right)^{q-p}=\left(\frac{p}{\lambda}\right)^{p} \frac{1}{q^{q}}
$$

Further if $l=\max \{p, q\}$, then

$$
N(r, a ; f \mid \leq 1)=\frac{l-2}{l} T(r, f)+S(r, f) .
$$

(ii) $f=\frac{(1-a) \gamma^{p}-1}{\frac{1-a}{a} \lambda \gamma^{-q}-1}$ and $g=\frac{\frac{1}{1-a} \gamma^{-p}-1}{\frac{a}{(1-) \lambda} \gamma^{q}-1}$, where $\gamma$ is a non-constant meromorphic function with $\bar{N}(r, 0 ; \gamma)+\bar{N}(r, \infty ; \gamma)=S(r, f)$, either $p=q=1$ or $p, q$ are mutually prime distinct positive integers and $\lambda(\neq 0)$ is a constant satisfying

$$
\left(\frac{q}{p+q}\right)^{q}=(-1)^{p}\left(\frac{\lambda(p+q)}{p}\right)^{p} .
$$

Further if $l=p+q$, then

$$
N(r, a ; f \mid \leq 1)=\frac{l-2}{l} T(r, f)+S(r, f) .
$$

However the possibility $(\mathrm{V})$ does not arise if $f, g$ share $(0, \infty),(1, \infty)$ and $(\infty, \infty)$.

\section{Lemmas}

In this section we present some necessary lemmas.

Lemma $2.1([4])$. Let $f, g$ share $(0,0),(1,0)$ and $(\infty, 0)$. Then

$$
T(r, f) \leq 3 T(r, g)+S(r, f) \text { and } T(r, g) \leq 3 T(r, f)+S(r, g) \text {. }
$$

Hence we see that $S(r, f)=S(r, g)=S(r)$. The following lemma can be proved in the line of Lemma 2 of [9].

Lemma $2.2([22])$. Let $f, g$ share $\left(0, k_{1}\right),\left(1, k_{2}\right),\left(\infty, k_{3}\right)$ and $f \not \equiv g$, where $k_{1} k_{2} k_{3}>k_{1}+k_{2}+k_{3}+2$. Then

$$
\bar{N}(r, b ; f \mid \geq 2)+\bar{N}(r, b ; g \mid \geq 2)=S(r)
$$

for $b=0,1, \infty$.

Using Lemma 2.2, the following the lemma can be proved in the line of Lemma 4 of [10].

Lemma 2.3. Let $f$ and $g$ be two distinct meromorphic functions sharing $\left(0, k_{1}\right),\left(1, k_{2}\right),\left(\infty, k_{3}\right)$, where $k_{1} k_{2} k_{3}>k_{1}+k_{2}+k_{3}+2$. If $\alpha=\frac{f-1}{g-1}$ and $h=\frac{g}{f}$, then $\bar{N}(r, b ; \alpha)+\bar{N}(r, b ; h)=S(r)$ for $b=0, \infty$. 
Lemma 2.4 ([13]). Let $f$ and $g$ be two distinct meromorphic functions sharing $(0,0),(1,0)$ and $(\infty, 0)$. If $f$ is a bilinear transformation of $g$, then $f$ and $g$ satisfy one of the following: (i) $f+g \equiv 1$, (ii) $(f-1)(g-1) \equiv 1$, (iii) $f g \equiv 1$, (iv) $f \equiv A g+1-A$, (v) $f \equiv A g$, (vi) $f(g+A-1) \equiv A g$, where $A(\neq 0,1, \infty)$ is a constant.

Lemma 2.5 ([15]). Let $f_{1}$ and $f_{2}$ be distinct non-constant meromorphic functions satisfying $\bar{N}\left(r, 0 ; f_{j}\right)+\bar{N}\left(r, \infty ; f_{j}\right)=S\left(r ; f_{1}, f_{2}\right)$ for $j=1,2$. If $f_{1}^{s} f_{2}^{t}-1$ is not identically zero for all integers $s, t(|s|+|t|>0)$, then for any positive number $\varepsilon$ we have

$$
N_{0}\left(r, 1 ; f_{1}, f_{2}\right) \leq \varepsilon T(r)+S\left(r ; f_{1}, f_{2}\right),
$$

where $N_{0}\left(r, 1 ; f_{1}, f_{2}\right)$ is the reduced counting function of the common 1-points of $f_{1}$ and $f_{2}, T(r)=T\left(r, f_{1}\right)+T\left(r, f_{2}\right)$ and $S\left(r ; f_{1}, f_{2}\right)=o\{T(r)\}$ as $r \rightarrow \infty$ except possibly for a set of finite linear measure.

Using Lemma 2.3 the following the lemma can be proved in the line of Lemma 3.9 of [12].

Lemma 2.6. Let $f$ and $g$ be two distinct non-constant meromorphic functions sharing $\left(0, k_{1}\right),\left(1, k_{2}\right),\left(\infty, k_{3}\right)$, where $k_{1} k_{2} k_{3}>k_{1}+k_{2}+k_{3}+2$. Suppose further that $f$ and $g$ satisfy one of the following:

(i) $(f-a)(g+a-1) \equiv a(1-a)$,

(ii) $f+(a-1) g \equiv a$,

(iii) $f \equiv a g$

for some $a(\not \equiv 0,1, \infty) \in \mathfrak{M}_{f, g}$. Then one of (I), (II) and (III) of Theorem 1.1 holds.

Lemma $2.7([17])$. Let $f$ be a non-constant meromorphic function and $R(f)=$ $\frac{P(f)}{Q(f)}$, where $P(f)=\sum_{k=0}^{p} a_{k} f^{k}$ and $Q(f)=\sum_{j=0}^{q} b_{j} f^{j}$ are two mutually prime polynomials in $f$. If the coefficients $a_{k}, b_{j}$ are small functions of $f$ and $a_{p} \not \equiv 0$, $b_{q} \not \equiv 0$, then

$$
T(r, R(f))=\max \{p, q\} T(r, f)+S(r, f) .
$$

Lemma 2.8 ([11]). Let $X$ and $Y$ be two meromorphic functions such that $X^{s} Y^{t} \equiv B$, where $B(\neq 0)$ is a constant and $s, t(\neq 0)$ are relatively prime integers. If $\gamma$ is a function defined by $\gamma^{t}=X$, then $\gamma$ is a single valued meromorphic function.

\section{Proof of Theorem 1.1}

Proof. First we suppose that $f$ is a bilinear transformation of $g$. Then by Lemma 2.4 we see that $f$ has two distinct Picard's exceptional values, say $a_{1}$ and $a_{2}$. If $a \equiv a_{1}$ or $a \equiv a_{2}$, then $N(r, a ; f \mid \leq 2) \neq T(r, f)+S(r, f)$ and so by Theorem $\mathrm{H}$ and Lemma 2.6 one of (I), (II) and (III) of the theorem holds. If 
$a \not \equiv a_{1}$ and $a \not \equiv a_{2}$, then by Nevanlinna's theorem on three small functions we get

$$
\begin{aligned}
T(r, f) & \leq \bar{N}(r, a ; f)+S(r, f) \\
& \leq \bar{N}(r, a ; f)+\frac{1}{2} N(r, a ; f \mid \geq 2)+S(r, f) \\
& \leq N(r, a ; f)+S(r, f) \\
& \leq T(r, f)+S(r, f) .
\end{aligned}
$$

Hence we obtain $N(r, a ; f \mid \geq 2)=S(r, f)$ and so $N(r, a ; f \mid \leq 1)=T(r, f)+$ $S(r, f)$, a contradiction.

Next we suppose that $f$ is not a bilinear transformation of $g$. We put $P=$ $\frac{\beta^{\prime}}{\beta}+\frac{a^{\prime}}{1-a}+\frac{a^{\prime}}{a}$ and $Q=\frac{\alpha^{\prime}}{\alpha}+\frac{a^{\prime}}{1-a}$, where $\beta=\alpha h$ and $\alpha, h$ are defined as in Lemma 2.3. Also we note that $\alpha, h$ and $\beta$ are non-constant.

We see that $T(r, \alpha) \leq T(r, f)+T(r, g)+O(1), T(r, h) \leq T(r, f)+T(r, g)+$ $O(1)$ and $T(r, \beta) \leq 2 T(r, f)+2 T(r, g)+O(1)$. Again since $f=\frac{1-\alpha}{1-\beta}$ and $g=\frac{1-\alpha^{-1}}{1-\beta^{-1}}$, it follows that $T(r, f) \leq T(r, \alpha)+T(r, \beta)+O(1)$ and $T(r, g) \leq$ $T(r, \alpha)+T(r, \beta)+O(1)$. Hence by Lemma 2.1 we get $S(r)=S(r ; f, g)=$ $S(r ; \alpha, \beta)$.

Now by Lemma 2.3 we obtain

$$
\begin{aligned}
\bar{N}(r, 0 ; \beta) & \leq \bar{N}(r, 0 ; \alpha)+\bar{N}(r, 0 ; h)=S(r), \\
\bar{N}(r, \infty ; \beta) & \leq \bar{N}(r, \infty ; \alpha)+\bar{N}(r, \infty ; h)=S(r), \\
T\left(r, \frac{\alpha^{\prime}}{\alpha}\right) & =m\left(r, \frac{\alpha^{\prime}}{\alpha}\right)+N\left(r, \frac{\alpha^{\prime}}{\alpha}\right) \\
& \leq \bar{N}(r, 0 ; \alpha)+\bar{N}(r, \infty ; \alpha)+S(r, \alpha) \\
& =S(r), \\
T\left(r, \frac{h^{\prime}}{h}\right) & =m\left(r, \frac{h^{\prime}}{h}\right)+N\left(r, \frac{h^{\prime}}{h}\right) \\
& \leq \bar{N}(r, 0 ; h)+\bar{N}(r, \infty ; h)+S(r, h) \\
T\left(r, \frac{\beta^{\prime}}{\beta}\right) & =T(r), \\
& \leq T\left(r, \frac{\alpha^{\prime}}{\alpha}+\frac{h^{\prime}}{h}\right)+T\left(r, \frac{h^{\prime}}{h}\right)+O(1) \\
& =S(r) .
\end{aligned}
$$

Hence $T(r, P)=S(r)$ and $T(r, Q)=S(r)$. Let $z_{0}$ be a multiple zero of $f-a$ which is not a zero of $P, Q$ and $P-Q$. Then

$$
f-a=\frac{(1-a)+a \beta-\alpha}{1-\beta}
$$


implies that

$$
1-a\left(z_{0}\right) \beta\left(z_{0}\right)-\alpha\left(z_{0}\right)=0
$$

and

$$
-a^{\prime}\left(z_{0}\right)+a^{\prime}\left(z_{0}\right) \beta\left(z_{0}\right)+a\left(z_{0}\right) \beta^{\prime}\left(z_{0}\right)=a\left(z_{0}\right) \beta^{\prime}\left(z_{0}\right)-\alpha^{\prime}\left(z_{0}\right)=0 .
$$

We put $f_{1}=\frac{1}{1-a} \cdot \frac{P-Q}{P} \alpha$ and $f_{2}=\frac{a}{1-a} \cdot \frac{P-Q}{Q} \beta$. Let $T(r, \alpha)=S(r)$. If $1-\alpha \equiv a$, then $f+(a-1) g \equiv a$, which is (ii) of Theorem H. So by Lemma 2.6 we obtain (II) of the theorem. If $1-\alpha \not \equiv a$, then $\alpha=\frac{f-1}{g-1}$ implies by Nevanlinna's theorem on three small functions

$$
\begin{aligned}
T(r, f) & \leq \bar{N}(r, 0 ; f)+\bar{N}(r, 1-\alpha ; f)+\bar{N}(r, a ; f)+S(r, f) \\
& =2 \bar{N}(r, 0 ; f)+\bar{N}(r, a ; f)+S(r, f) \\
& \leq 2 N(r, 1 ; \alpha)+\bar{N}(r, a ; f)+S(r, f) \\
& =\bar{N}(r, a ; f)+S(r, f) .
\end{aligned}
$$

This implies that $N(r, a ; f \mid \leq 1)=T(r, f)+S(r, f)$, a contradiction.

Let $T(r, \beta)=S(r)$. If $\frac{1}{1-\beta} \equiv a$, then $(f-a)(g+a-1) \equiv a(1-a)$, which is (i) of Theorem H. So by Lemma 2.6 we obtain (I) of the theorem. If $\frac{1}{1-\beta} \not \equiv a$, then $\beta=\frac{1-\frac{1}{f}}{1-\frac{1}{g}}$ implies by Nevanlinna's theorem on three small functions $T(r, f) \leq \bar{N}(r, a ; f)+S(r, f)$ and so $N(r, a ; f \mid \leq 1)=T(r, f)+S(r, f)$, a contradiction.

So we suppose that $T(r, \alpha) \neq S(r)$ and $T(r, \beta) \neq S(r)$. Therefore $f_{1}$ and $f_{2}$ are non-constant. Clearly $S(r)=S(r ; f, g)=S(r ; \alpha, \beta)=S\left(r ; f_{1}, f_{2}\right)$. Again for $b=0, \infty$ we get

$$
\bar{N}\left(r, b ; f_{1}\right) \leq \bar{N}(r, b ; \alpha)+T\left(r, \frac{P-Q}{(1-a) P}\right)=S\left(r ; f_{1}, f_{2}\right)
$$

and

$$
\bar{N}\left(r, b ; f_{2}\right) \leq \bar{N}(r, b ; \beta)+T\left(r, \frac{a(P-Q)}{(1-a) Q}\right)=S\left(r ; f_{1}, f_{2}\right) .
$$

Also from (3.1) and (3.2) we see that

$$
\bar{N}(r, a ; f \mid \geq 2) \leq N_{0}\left(r, 1 ; f_{1}, f_{2}\right)+S\left(r ; f_{1}, f_{2}\right) .
$$

We first suppose that $f_{1}^{s} f_{2}^{t} \not \equiv 1$ for any two integers $s$ and $t(|s|+|t|>0)$. Then by Lemma 2.1 and Lemma 2.5 we get from $(3.3)$ that $\bar{N}(r, a ; f \mid \geq 2) \leq$ $\varepsilon T(r, f)+S(r, f)$, where $\varepsilon(>0)$ is arbitrary. If $T(r, f)=N(r, a ; f \mid \leq 2)+$ $S(r, f)$, then we obtain

$$
\begin{aligned}
T(r, f) & =N(r, a ; f \mid \leq 2)+S(r, f) \\
& \leq N(r, a ; f \mid \leq 1)+\varepsilon T(r, f)+S(r, f) .
\end{aligned}
$$

This shows that

$$
1-\varepsilon \leq \liminf _{r \rightarrow \infty, r \notin E} \frac{N(r, a ; f \mid \leq 1)}{T(r, f)} \leq \limsup _{r \rightarrow \infty, r \notin E} \frac{N(r, a ; f \mid \leq 1)}{T(r, f)} \leq 1,
$$


where $E$ is a set of finite linear measure.

Since $\varepsilon(>0)$ is arbitrary, we get

$$
\lim _{r \rightarrow \infty, r \notin E} \frac{N(r, a ; f \mid \leq 1)}{T(r, f)}=1,
$$

which is a contradiction. So $N(r, a ; f \mid \leq 2) \neq T(r, f)+S(r, f)$ and (I), (II) or (III) of the theorem follows from Theorem $\mathrm{H}$ and Lemma 2.6.

We now suppose that there exist two integers $s$ and $t(|s|+|t|>0)$ such that

$$
f_{1}^{s} f_{2}^{t} \equiv 1
$$

Since $f_{1}$ and $f_{2}$ are non-constant, we see that $s \neq 0$ and $t \neq 0$. Without loss of generality we choose $t>0$. From (3.4) we obtain

$$
\alpha^{s} \beta^{t} \equiv\left(\frac{1-a}{1-\frac{Q}{P}}\right)^{s}\left(\frac{1-a}{a\left(\frac{P}{Q}-1\right)}\right)^{t} .
$$

By logarithmic differentiation we get

$$
s Q+t P \equiv \frac{s Q+t P}{Q\left(1-\frac{Q}{P}\right)}\left(\frac{Q}{P}\right)^{\prime} .
$$

Let $s Q+t P \not \equiv 0$. Then we obtain

$$
\frac{\alpha^{\prime}}{\alpha}+\frac{a^{\prime}}{1-a} \equiv \frac{\left(\frac{Q}{P}\right)^{\prime}}{1-\frac{Q}{P}} .
$$

On integration we get $\alpha \equiv \frac{A(1-a)}{1-\frac{Q}{P}}$, where $A(\neq 0)$ is a constant. This implies $T(r, \alpha)=S(r)$, a contradiction. Therefore

$$
s Q+t P \equiv 0 .
$$

If $s=t$, then in view of (3.5) we can put $s=t=1$. Again if $s \neq t$, then in view of (3.5) we can choose $s$ and $t$ to be mutually prime. From (3.5) we get on integration

$$
\alpha^{s} \beta^{t} \equiv B \frac{(1-a)^{s+t}}{a^{t}}
$$

where $B(\neq 0)$ is a constant

If $s+t=0$, then from (3.6) we see that $T(r, h)=S(r)$. If $h \not \equiv a^{-1}$, then by Nevanlinna's theorem on three small functions we get

$$
T(r, f) \leq \bar{N}(r, a ; f)+S(r, f) .
$$

This shows that $N(r, a ; f \mid \leq 1)=T(r, f)+S(r, f)$, a contradiction. Hence $h \equiv a^{-1}$ and so $f \equiv a g$, which is (iii) of Theorem H. So by Lemma 2.6 we obtain (III) of the theorem. 
We now suppose that $s+t \neq 0$. If $a$ is a constant, then from (3.6) we see that $f, g$ share $(0, \infty),(1, \infty)$ and $(\infty, \infty)$. So by Theorem $\mathrm{C}$ we obtain (IV) of the theorem.

Now we suppose that $a$ is non-constant. However we note that this possibility does not arise when $f, g$ share $(0, \infty),(1, \infty)$ and $(\infty, \infty)$. For, then $\alpha$ and $\beta$ become exponential functions and so from (3.6) we see that $0,1, \infty$ are Picard exceptional values of $a$, which is impossible.

We put $X=\frac{\alpha}{1-a}, Y=\frac{a}{1-a} \beta$ and $\gamma^{t}=X$. Since from (3.6) we get $X^{s} Y^{t} \equiv$ $B$, by Lemma 2.8 we see that $\gamma$ is a single valued meromorphic function. Also by Lemma 2.3 we get $\bar{N}(r, 0 ; \gamma)+\bar{N}(r, \infty ; \gamma)=S(r)$. Then putting $\lambda^{t}=B$ we obtain

$$
f=\frac{\alpha-1}{\beta-1}=\frac{(1-a) \gamma^{t}-1}{\frac{1-a}{a} \lambda \gamma^{-s}-1} \text { and } g=\frac{\alpha^{-1}-1}{\beta^{-1}-1}=\frac{\frac{1}{1-a} \gamma^{-t}-1}{\frac{a}{(1-a) \lambda} \gamma^{s}-1}
$$

We consider the following two cases.

Case I. Let $t>0$ and $s<0$. We put $p=t$ and $q=-s$. Then $p$ and $q$ are two distinct mutually prime positive integers and

$$
f=\frac{(1-a) \gamma^{p}-1}{\frac{1-a}{a} \lambda \gamma^{q}-1} \text { and } g=\frac{\frac{1}{1-a} \gamma^{-p}-1}{\frac{a}{(1-a) \lambda} \gamma^{-q}-1} .
$$

Hence

$$
f-a=(1-a) \frac{\gamma^{p}-\lambda \gamma^{q}-1}{\frac{1-a}{a} \lambda \gamma^{q}-1}
$$

If the equation

$$
w^{p}-\lambda w^{q}-1=0
$$

has only simple roots, say $\mu_{1}, \mu_{2}, \ldots, \mu_{l}$, where $l=\max \{p, q\} \geq 2$, then by Lemma 2.7 we get from $(3.7)$

$$
\begin{aligned}
N(r, a ; f \mid \leq 1) & =\sum_{j=1}^{l} \bar{N}\left(r, \mu_{j} ; \gamma\right)+S(r, \gamma) \\
& =l T(r, \gamma)+S(r, \gamma) \\
& =T(r, f)+S(r, f),
\end{aligned}
$$

a contradiction. So equation (3.8) has at least one multiple root, say $\mu$. Then we get

$$
\mu^{p}-\lambda \mu^{q}-1=0
$$

and

$$
p \mu^{p-1}-\lambda q \mu^{q-1}=0 .
$$


Since $\mu \neq 0$, from (3.9) and (3.10) we get

$$
\mu^{p}=\frac{q}{q-p} \text { and } \mu^{q}=\frac{p}{\lambda(q-p)} .
$$

Eliminating $\mu$ we obtain

$$
\left(\frac{1}{q-p}\right)^{q-p}=\left(\frac{p}{\lambda}\right)^{p} \frac{1}{q^{q}}
$$

If $\mu$ is a root of (3.8) with multiplicity $\geq 3$, then

$$
p(p-1) \mu^{p-2}-\lambda q(q-1) \mu^{q-2}=0 .
$$

From (3.10) and (3.12) we get $p=q$, a contradiction. So the equation (3.8) does not have any root of multiplicity $\geq 3$.

If $\mu_{1}$ and $\mu_{2}$ be two double roots of (3.8), then by (3.11) we get $\mu_{1}^{p}=\mu_{2}^{p}$ and $\mu_{1}^{q}=\mu_{2}^{q}$. Since $p$ and $q$ are mutually prime, there exist two integers $x$ and $y$ such that $x p+y q=1$. So

$$
\mu_{1}=\mu_{1}^{x p+y q}=\left(\mu_{1}^{p}\right)^{x}\left(\mu_{1}^{q}\right)^{y}=\left(\mu_{2}^{p}\right)^{x}\left(\mu_{2}^{q}\right)^{y}=\mu_{2}^{x p+y q}=\mu_{2} .
$$

So the equation (3.8) has only one double root and $l-2$ simple roots. Therefore by Lemma 2.7 we get

$$
N(r, a ; f \mid \leq 1)=\frac{l-2}{l} T(r, f)+S(r, f) .
$$

Case II. Let $t>0$ and $s>0$. Putting $p=t$ and $q=s$ we get

$$
f=\frac{(1-a) \gamma^{p}-1}{\frac{1-a}{a} \lambda \gamma^{-q}-1} \text { and } g=\frac{\frac{1}{1-a} \gamma^{-p}-1}{\frac{a}{(1-a) \lambda} \gamma^{q}-1},
$$

where either $p=q=1$ or $p, q$ are mutually prime distinct positive integers.

Hence

$$
f-a=(1-a) \frac{\gamma^{p+q}-\gamma^{q}-\lambda}{\frac{1-a}{a} \lambda-\gamma^{q}} .
$$

Since $N(r, a ; f \mid \leq 1) \neq T(r, f)+S(r, f)$, we see that the equation

$$
w^{p+q}-w^{q}-\lambda=0
$$

must have one multiple root, say $\nu$. Then we get

$$
\nu^{p+q}-\nu^{q}-\lambda=0 \text { and }(p+q) \nu^{p+q-1}-q \nu^{q-1}=0 .
$$

Since $\nu \neq 0$, this implies

$$
\nu^{p}=\frac{q}{p+q} \text { and } \nu^{q}=-\frac{\lambda(p+q)}{p} .
$$

Eliminating $\nu$ we obtain

$$
\left(\frac{q}{p+q}\right)^{q}=(-1)^{p}\left(\frac{\lambda(p+q)}{p}\right)^{p} .
$$


Proceeding as Case I we can verify that the equation (3.14) has only one double root and $p+q-2$ simple roots. Therefore by Lemma 2.7 we get

$$
N(r, a ; f \mid \leq 1)=\frac{l-2}{l} T(r, f)+S(r, f),
$$

where $l=p+q$. This proves the theorem.

\section{A result of X. M. Li and H. X. Yi}

In 2004 improving a result of P. Li [14], X. M. Li and H. X. Yi [16] proved the following result.

Theorem I ([16]). Let $f$ and $g$ be two distinct non-constant meromorphic functions sharing 0, 1 and $\infty$ CM. If there exists a finite complex number $a(\neq 0,1)$ such that $a$ is not a Picard's exceptional value of $f$ and

$$
N(r, a ; f \mid \leq 1) \leq u T(r, f)+S(r, f),
$$

where $0<u<\frac{1}{3}$, then $N(r, a ; f \mid \leq 1) \equiv 0$ and $f, g$ assume one of the following forms:

(i) $f=\frac{\gamma^{3}-1}{\gamma-1}$ and $g=\frac{\gamma^{-3}-1}{\gamma^{-1}-1}$ with $a=\frac{3}{4}$;

(ii) $f=\frac{\gamma^{3}-1}{\gamma^{2}-1}$ and $g=\frac{\gamma^{-3}-1}{\gamma^{-2}-1}$ with $a=-3$;

(iii) $f=\frac{\gamma-1}{\gamma^{3}-1}$ and $g=\frac{\gamma^{-1}-1}{\gamma^{-3}-1}$ with $a=\frac{4}{3}$;

(iv) $f=\frac{\gamma^{2}-1}{\gamma^{3}-1}$ and $g=\frac{\gamma^{-2}-1}{\gamma^{-3}-1}$ with $a=-\frac{1}{3}$;

(v) $f=\frac{\gamma^{2}-1}{\gamma^{-1}-1}$ and $g=\frac{\gamma^{-2}-1}{\gamma-1}$ with $a=\frac{1}{4}$;

(vi) $f=\frac{\gamma-1}{\gamma^{-2}-1}$ and $g=\frac{\gamma^{-1}-1}{\gamma^{2}-1}$ with $a=4$;

(vii) $f=\frac{\gamma^{2}-1}{\lambda \gamma-1}$ and $g=\frac{\gamma^{-2}-1}{\frac{1}{\lambda} \gamma^{-1}-1}$ with $\lambda^{2} \neq 1$ and $a^{2} \lambda^{2}=4(a-1)$;

(viii) $f=\frac{\gamma-1}{\lambda \gamma^{2}-1}$ and $g=\frac{\gamma^{-1}-1}{\frac{1}{\lambda} \gamma^{-2}-1}$ with $\lambda \neq 1$ and $4 a(1-a) \lambda=1$;

(ix) $f=\frac{\gamma-1}{\lambda \gamma^{-1}-1}$ and $g=\frac{\gamma^{-1}-1}{\frac{1}{\lambda} \gamma-1}$ with $\lambda \neq 1$ and $(1-a)^{2}+4 a \lambda=0$, where $\gamma$ is a non-constant exponential function.

Considering $f=e^{3 z}+e^{2 z}+e^{z}+1, g=e^{-3 z}+e^{-2 z}+e^{-z}+1$ and $a=\frac{20+4 \sqrt{2} i}{27}$ (see [14] and [16]) one can verify that in the hypothesis (4.1) it is not possible to choose $u=\frac{1}{3}$. So it is a natural query to investigate the forms of the functions 
$f$ and $g$ when we put $u=\frac{1}{3}$ in (4.1). In the following theorem we answer this query under a more general setting.

Theorem 4.1. Let $f$ and $g$ be two distinct non-constant meromorphic functions sharing $\left(0, k_{1}\right),\left(1, k_{2}\right),\left(\infty, k_{3}\right)$, where $k_{1} k_{2} k_{3}>k_{1}+k_{2}+k_{3}+2$. If

$$
N(r, a ; f \mid \leq 1)=\frac{1}{3} T(r, f)+S(r, f)
$$

for some $a(\not \equiv 0,1, \infty) \in \mathfrak{M}_{f, g}$, then one of the following holds:

(I) If a is a constant, then there exists a non-constant exponential function $\mu$ such that $f$ and $g$ are one of the following forms:

(i) $f=\frac{\mu^{3}-1}{\lambda \mu-1}$ and $g=\frac{\mu^{-3}-1}{\frac{1}{\lambda} \mu^{-1}-1}$ with $\lambda^{3} \neq 1$ and $\left(\frac{a-1}{2}\right)^{2}=\left(\frac{a \lambda}{3}\right)^{3}$;

(ii) $f=\frac{\mu^{3}-1}{\lambda \mu^{2}-1}$ and $g=\frac{\mu^{-3}-1}{\frac{1}{\lambda} \mu^{-2}-1}$ with $\lambda^{3} \neq 1$ and $\frac{a-1}{4}=\left(\frac{a \lambda}{3}\right)^{3}$;

(iii) $f=\frac{\mu-1}{\lambda \mu^{3}-1}$ and $g=\frac{\mu^{-1}-1}{\frac{1}{\lambda} \mu^{-3}-1}$ with $\lambda \neq 1$ and $\left(\frac{2}{1-a}\right)^{2}=27 a \lambda$;

(iv) $f=\frac{\mu^{2}-1}{\lambda \mu^{3}-1}$ and $g=\frac{\mu^{-2}-1}{\frac{1}{\lambda} \mu^{-3}-1}$ with $\lambda^{2} \neq 1$ and $\frac{4}{1-a}=27 a^{2} \lambda^{2}$;

(v) $f=\frac{\mu^{4}-1}{\mu-1}$ and $g=\frac{\mu^{-4}-1}{\mu^{-1}-1}$ with $a \neq 4$ and $\left(\frac{a-1}{3}\right)^{3}=\left(\frac{a}{4}\right)^{4}$;

(vi) $f=\frac{\mu^{4}-1}{\mu^{3}-1}$ and $g=\frac{\mu^{-4}-1}{\mu^{-3}-1}$ with $a \neq \frac{4}{3}$ and $256(a-1)=27 a^{4}$;

(vii) $f=\frac{\mu-1}{\mu^{4}-1}$ and $g=\frac{\mu^{-1}-1}{\mu^{-4}-1}$ with $a \neq \frac{1}{4}$ and $\left(\frac{3}{1-a}\right)^{3}=256 a$;

(viii) $f=\frac{\mu^{3}-1}{\mu^{4}-1}$ and $g=\frac{\mu^{-3}-1}{\mu^{-4}-1}$ with $a \neq \frac{3}{4}$ and $\frac{1}{1-a}=256\left(\frac{a}{3}\right)^{3}$;

(ix) $f=\frac{\mu^{2}-1}{\lambda \mu^{-1}-1}$ and $g=\frac{\mu^{-2}-1}{\frac{1}{\lambda} \mu-1}$ with $\lambda^{2} \neq 1$ and $\frac{4(1-a)}{3}=\left(\frac{3 a \lambda}{a-1}\right)^{2}$;

(x) $f=\frac{\mu-1}{\lambda \mu^{-2}-1}$ and $g=\frac{\mu^{-1}-1}{\frac{1}{\lambda} \mu^{2}-1}$ with $\lambda \neq 1$ and $\left(\frac{2-2 a}{3}\right)^{2}=\frac{3 a \lambda}{a-1}$;

(xi) $f=\frac{\mu^{3}-1}{\mu^{-1}-1}$ and $g=\frac{\mu^{-3}-1}{\mu-1}$ with $a \neq-3$ and $\frac{1-a}{4}=\left(\frac{4 a}{3 a-3}\right)^{3}$;

(xii) $f=\frac{\mu-1}{\mu^{-3}-1}$ and $g=\frac{\mu^{-1}-1}{\mu^{3}-1}$ with $a \neq-\frac{1}{3}$ and $\left(\frac{3-3 a}{4}\right)^{3}=\frac{4 a}{a-1}$.

(II) If a is non-constant, then there exists a non-constant meromorphic function $\mu$ with $\bar{N}(r, 0 ; \mu)+\bar{N}(r, \infty ; \mu)=S(r, f)$ such that $f$ and $g$ are one of the following forms: 
(xiii) $f=\frac{(1-a) \mu^{3}-1}{\frac{1-a}{a} \lambda \mu-1}$ and $g=\frac{\frac{1}{1-a} \mu^{-3}-1}{\frac{a}{(1-a) \lambda} \mu^{-1}-1}$ with $\lambda^{3}=\frac{27}{4}$;

(xiv) $f=\frac{(1-a) \mu^{3}-1}{\frac{1-a}{a} \lambda \mu^{2}-1}$ and $g=\frac{\frac{1}{1-a} \mu^{-3}-1}{\frac{a}{(1-a) \lambda} \mu^{-2}-1}$ with $\lambda^{3}=-\frac{27}{4}$;

(xv) $f=\frac{(1-a) \mu-1}{\frac{1-a}{a} \lambda \mu^{3}-1}$ and $g=\frac{\frac{1}{1-a} \mu^{-1}-1}{\frac{a}{(1-a) \lambda} \mu^{-3}-1}$ with $\lambda=\frac{4}{27}$;

(xvi) $f=\frac{(1-a) \mu^{2}-1}{\frac{1-a}{a} \lambda \mu^{3}-1}$ and $g=\frac{\frac{1}{1-a} \mu^{-2}-1}{\frac{a}{(1-a) \lambda} \mu^{-3}-1}$ with $\lambda^{2}=\frac{4}{27}$;

(xvii) $f=\frac{(1-a) \mu^{2}-1}{\frac{1-a}{a} \lambda \mu^{-1}-1}$ and $g=\frac{\frac{1}{1-a} \mu^{-2}-1}{\frac{a}{(1-a) \lambda} \mu-1}$ with $\lambda^{2}=\frac{4}{27}$;

(xviii) $f=\frac{(1-a) \mu-1}{\frac{1-a}{a} \lambda \mu^{-2}-1}$ and $g=\frac{\frac{1}{1-a} \mu^{-1}-1}{\frac{a}{(1-a) \lambda} \mu^{2}-1}$ with $\lambda=-\frac{4}{27}$.

Further if $f, g$ share $(0, \infty),(1, \infty)$ and $(\infty, \infty)$, then possibility (II) does not arise.

Proof. By the given condition we see that only the possibilities (IV) and (V) of Theorem 1.1 will occur.

If the possibility (IV) occurs then putting $k=3$ and $\gamma=\mu$ we get the possibility (I) of the theorem.

Now we suppose that the possibility (V) of Theorem 1.1 occurs. By (4.2) we see that either $l=\max \{p, q\}=3$ or $l=p+q=3$.

If $l=\max \{p, q\}=3$, then

1. for $p=3, q=1$ and $\mu=\gamma$ we obtain (xiii) of the theorem,

2. $p=3, q=2$ and $\mu=\gamma$ we obtain (xiv) of the theorem,

3. for $p=1, q=3$ and $\mu=\gamma$ we obtain (xv) of the theorem,

4. for $p=2, q=3$ and $\mu=\gamma$ we obtain (xvi) of the theorem.

Again if $l=p+q=3$, then

5. for $p=2, q=1$ and $\mu=\gamma$ we obtain (xvii) of the theorem,

6. for $p=1, q=2$ and $\mu=\gamma$ we obtain (xviii) of the theorem.

Also it is clear from Theorem 1.1 that the possibility (II) does not occur if $f$, $g$ share $(0, \infty),(1, \infty)$ and $(\infty, \infty)$. This proves the theorem.

\section{References}

[1] T. C. Alzahary, Small functions and weighted sharing three values, Complex Var. Theory Appl. 50 (2005), no. 15, 1105-1124.

[2] _ Small functions of meromorphic functions sharing three values with finite weights, Indian J. Pure Appl. Math. 38 (2007), no. 4, 305-316.

[3] T. C. Alzahary and H. X. Yi, Weighted sharing three values and uniqueness of meromorphic functions, J. Math. Anal. Appl. 295 (2004), no. 1, 247-257.

[4] G. G. Gundersen, Meromorphic functions that share three or four values, J. London Math. Soc. (2) 20 (1979), no. 3, 457-466. 
[5] W. K. Hayman, Meromorphic Functions, The Clarendon Press, Oxford, 1964.

[6] I. Lahiri, Weighted sharing of three values and uniqueness of meromorphic functions, Kodai Math. J. 24 (2001), no. 3, 421-435.

[7] _ Weighted sharing and uniqueness of meromorphic functions, Nagoya Math. J. 161 (2001), 193-206.

[8] - Weighted value sharing and uniqueness of meromorphic functions, Complex Variables Theory Appl. 46 (2001), no. 3, 241-253.

[9] _ On a result of Ozawa concerning uniqueness of meromorphic functions. II, J. Math. Anal. Appl. 283 (2003), no. 1, 66-76.

[10] _ Sharing three values with small weights, Hokkaido Math. J. 36 (2007), no. 1, $129-142$.

[11] Weighted sharing of three values by meromorphic functions, Hokkaido Math. J. 37 (2008), no. 1, 41-58.

[12] I. Lahiri and N. Mandal, Small functions and uniqueness of meromorphic functions, J. Math. Anal. Appl. 340 (2008), no. 2, 780-792.

[13] I. Lahiri and A. Sarkar, On a uniqueness theorem of Tohge, Arch. Math. (Basel) 84 (2005), no. 5, 461-469.

[14] P. Li, Meromorphic functions sharing three values or sets CM, Kodai Math. J. 21 (1998), no. 2, 138-152.

[15] P. Li and C. C. Yang, On the characteristics of meromorphic functions that share three values CM, J. Math. Anal. Appl. 220 (1998), no. 1, 132-145.

[16] X. M. Li and H. X. Yi, Meromorphic functions sharing three values, J. Math. Soc. Japan 56 (2004), no. 1, 147-167.

[17] A. Z. Mohonko, The Nevanlinna characteristics of certain meromorphic functions, Teor. Funkcií Funkcional. Anal. i Priloìen. No. 14 (1971), 83-87.

[18] H. Ueda, Unicity theorems for meromorphic or entire functions, Kodai Math. J. 3 (1980), no. 3, 457-471.

[19] S. Z. Ye, Uniqueness of meromorphic functions that share three values, Kodai Math. J. 15 (1992), no. 2, 236-243.

[20] H. X. Yi, Meromorphic functions that share three values, Chinese Ann. Math. Ser. A 9 (1988), no. 4, 434-439.

[21] Unicity theorems for meromorphic functions that share three values, Kodai Math. J. 18 (1995), no. 2, 300-314.

[22] _ Meromorphic functions with weighted sharing of three values, Complex Var. Theory Appl. 50 (2005), no. 12, 923-934.

INDRAJIT LAHIRI

Department of Mathematics

UNIVERSITY OF KALYANI

West Bengal 741235, India

E-mail address: indr9431@dataone.in

PUlaK SAHOO

Department of Mathematics

Silda Chandra Sekhar College

Silda, West Bengal 721515, India

E-mail address: sahoopulak@yahoo.com 